Pacific Journal of Mathematics

DUAL GROUPS OF VECTOR SPACES

William Charles Waterhous 


\section{DUAL GROUPS OF VECTOR SPACES}

\section{William C. Waterhouse}

Let $E$ be a topological vector space over a field $K$ having a nontrivial absolute value. Let $E^{\prime}$ be the dual space of continuous linear maps $E \rightarrow K$, and $\hat{E}$ the dual group of continuous characters $E \rightarrow R / Z$. $\hat{E}$ is a vector space over $K$ by $(a \varphi)(x)=\varphi(a x)$, and composition with a nonzero character of $K$ is a linear map of $E^{\prime}$ into $\hat{E}$. This map is always an isomorphism if $K$ is locally compact, while if $K$ is not locally compact it is never an isomorphism unless $\hat{E}=0$. When $K$ is locally compact, $E^{\prime}$ is in addition topologically isomorphic to $\hat{E}$ if each is given its topology of uniform convergence on compact sets. This leads to conditions on $E$ which imply that $E$ is topologically isomorphic to $(\hat{E})^{\wedge}$.

Theorem 1. Let $K$ be a field with absolute value. Then $\hat{K}$ is one-dimensional over $K$ if and only if $K$ is locally compact.

Proof. The sufficiency of local compactness is fairly well known (cf. [4, p. 92-3] for the characteristic zero case). To prove it, one takes a nonzero character $\pi$ of $K$ and considers the subspace $K \pi$ of $\hat{K}$. It is easy to check that $a \mapsto a \pi$ is a bicontinuous linear map, so $K \pi$ is complete and hence closed in $\hat{K}$. On the other hand, $K \pi$ separates the points of $K$, so by Pontrjagin duality it is dense in $\hat{K}$. Thus $\hat{K}=K \pi$.

Suppose conversely that $\hat{K}$ is one-dimensional, and choose a nonzero $\pi$ in $\hat{K}$. The completion of $K$ will again be a field, say $L$, and $\pi$ extends to a character of $L$. Then every $a \in L$ gives a character $a \pi$ of $L$. If $a \neq b$, then $a-b$ is invertible, and so $\pi((a-b) c)$ cannot be zero for all $c$. Thus no two of the characters $a \pi$ are equal, and hence no two can agree on the dense set $K$. This contradicts onedimensionality of $\hat{K}$ unless $K=L$, and we conclude that $K$ must be complete. Hence if $K$ is archimedean, it is locally compact.

We now assume that $K$ is nonarchimedean. Let $A=\{x:|x| \leqq 1\}$, $M=\{x:|x|<1\}$. Let $\pi$ be a character of the discrete group $A / M$ with $\pi(1) \neq 0$; we extend $\pi$ to a character of the discrete group $K / M$ and interpret it as an element of $\hat{K}$. Let $c>1$ be an element of the value group, and consider the group $G_{c} / M$, where $G_{c}=\{x:|x| \leqq c\}$. All characters of this discrete group extend to characters of $K$ vanishing on $M$, and by one-dimensionality they all come from multiples of $\pi$.

Now if $a \in A$, then $a M \subset M$, so $a \pi$ vanishes on $M$; conversely, if $a \pi$ vanishes on $M$, then $1 / a \notin M$ and $a \in A$. Similarly, $a \pi$ vanishes 
on $G_{c}$ if and only if $|a|<1 / c$. Thus the dual group of the discrete abelian group $G_{c} / M$ is (algebraically) isomorphic to $A /\{a:|a|<1 / c\}$, which is isomorphic to $G_{c} / M$ itself under multiplication by an element of absolute value $c$. A theorem of Kakutani [3, p. 396-7] shows that an infinite discrete abelian group has a dual group of strictly larger cardinality; hence $G_{c} / M$ must be finite. This implies both that $A / M$ is finite and that the value group is discrete; since $K$ has these two properties and is complete, it is locally compact [1, p. 119].

Corollary. Suppose $K$ is not locally compact. Let $E$ be a topological vector space over $K$ with $\hat{E} \neq 0$. For any $\pi \in \hat{K}$, the map $E^{\prime} \rightarrow \hat{E}$ given by composition with $\pi$ fails to be surjective.

Proof. If $E^{\prime}=0$ or $\pi=0$, the statement is obvious. Suppose then there is a $0 \neq f \in E^{\prime}$, and choose an $x \in E$ with $f(x) \neq 0$. The subspace $K x$ is topologically isomorphic to $K$, so its dual space is one-dimensional and is generated by the restriction of $f$. Hence all elements in $\hat{E}$ coming from $E^{\prime}$ restrict to multiples of $\pi \circ f$ on $K x$. If $\tau$ is a character of $K$ not a multiple of $\pi$, then $\tau \circ f \in \hat{E}$ is not in the image of $E^{\prime}$.

REMARK. A topological field $K$ is called locally retrobounded if for every pair of neighborhoods $U, V$ of zero there is an $a \neq 0$ in $K$ such that $a\left\{x^{-1}: x \notin V\right\} \subset U$; for example, an ordered field is locally retrobounded in its order topology. Every such field admits either an absolute value or a valuation which defines its topology $[1, \S 5$, Exerc. 2]. The proof of Theorem 1 works equally well for a valuation into any ordered abelian group, and hence Theorem 1 and its corollary hold for all locally retrobounded fields.

THEOREM 2. Suppose $K$ is locally compact, $0 \neq \pi \in \hat{K}$. Let $E$ be a topological vector space over $K$. Then the map $E^{\prime} \rightarrow \hat{E}$ given by $f \mapsto \pi \circ f$ is a vector space isomorphism. It is a homeomorphism if $E^{\prime}$ and $\hat{E}$ have their topologies of uniform convergence on compact sets.

Proof. If $0 \neq f$, then $f(E)=K$, so $\pi \circ f \neq 0$; thus the map is injective. Now let $\varphi \in \hat{E}$. For each $x \in E$ there is a unique linear functional on $K x$ inducing $\varphi \mid K x$, since $K x \cong K$ and $\hat{K}$ is onedimensional. We define $f(x)$ to be this functional evaluated at $x$; this gives us a homogeneous function $f: E \rightarrow K$. For any $x, y \in E$ and $a \in K$, we have

$$
\begin{aligned}
0 & =\varphi(a x)+\varphi(a y)-\varphi(a x+a y)=\pi f(a x)+\pi f(a y)-\pi f(a x+a y) \\
& =\pi[f(a x)+f(a y)-f(a x+a y)]=\pi(a[f(x)+f(y)-f(x+y)]) ;
\end{aligned}
$$


hence $f(x)+f(y)-f(x+y)=0$, and $f$ is linear. If finally $f$ were not continuous, then $f^{-1}(a)$ would be dense in $E$ for every $a \in K$. Hence $f(U)=K$ for any neighborhood $U$ of zero, so $\varphi(U)=\pi \circ f(U)=$ $\pi(K)$ for all such $U$ and $\varphi$ would not be continuous.

Now the map $E^{\prime} \rightarrow \widehat{E}$ is an isomorphism, and it is obviously continuous; we need only prove it is open. The map $K^{\prime} \rightarrow \hat{K}$ is a homeomorphism, since (as we noted in the proof of Theorem 1 ) $\hat{K} \cong K$. Hence, given any neighborhood $U$ of zero in $K$, we can find an open $V$ and a compact set $B$ such that, for $g$ in $K^{\prime}, \pi \circ g(B) \subset V$ implies $g(a) \in U$ for $|a| \leqq 1$. But if $C$ is any compact set in $E, B C$ will again be compact. It is easy to see then that if $f \in E^{\prime}$ and $\pi \circ f(B C) \subset V$, then $f(C) \subset U$; this means that $E^{\prime} \rightarrow \hat{E}$ is open.

Let $K$ again be locally compact, and let $E$ be a locally convex topological vector space over $K$. (In the archimedean case, the requisite theory is standard, cf. [2]; van Tiel has shown that exactly the same theory holds in the nonarchimedean case [6].) In view of Theorem 2, we identify $E^{\prime}$ and $\hat{E}$ furnished with the topology of uniform convergence on compact sets.

THEOREM 3. If $E$ is quasi-complete and barrelled, then $E$ is topologically isomorphic to $(\hat{E})^{\wedge}$.

Proof. Since $E$ is locally convex, the map $E \rightarrow(\hat{E})^{\wedge}$ is injective. Since $E$ is quasi-complete, the closed convex hull of a compact set is compact; thus the topology on $\hat{E}$ is that of uniform convergence on convex compact sets. This is weaker than the Mackey topology, and hence the map $E \rightarrow(\hat{E})^{\wedge}$ is bijective.

If $S$ is a compact balanced set in $\hat{E}$, then its polar $S^{\circ}$ is a barrel in $E$, and hence is a neighborhood of 0 in $E$. These polars are a neighborhood basis at 0 in $(\hat{E})^{\wedge}$, so the map $E \rightarrow(\hat{E})^{\wedge}$ is continuous.

Finally, if $U$ is a neighborhood of 0 in $E, U^{\circ}$ is equicontinuous and therefore compact in $\hat{E}$; hence $U^{\circ \circ}$ is a neighborhood of 0 in $(\hat{E})^{\wedge}$. But $E$ has a neighborhood basis at 0 consisting of closed absolutely convex sets $U$, and for them $U=U^{\circ \circ}$. Thus the map is open.

As particular cases of Theorem 3, we get

COROLlaRY. If $E$ is either complete and metrizable or reflexive, $E$ is topologically isomorphic to $(\hat{E})^{\wedge}$.

For the real and complex fields, Theorem 2 and these two cases of Theorem 3 were proved by M. F. Smith [5]. 


\section{REFERENCES}

1. N. Bourbaki, Algèbre Commutative, Hermann, Paris, 1964.

2. N. Bourbaki, Espaces Vectoriels Topologiques, Hermann, Paris, 1964.

3. E. Hewitt and K. Ross, Abstract Harmonic Analysis, Vol. 1, Springer-Verlag, Berlin, 1963

4. S. Lang, Algebraic Numbers, Addison-Wesley, Reading, Mass., 1964.

5. M. F. Smith, The Pontrjagin duality theorem in linear spaces, Ann. of Math. (2) 56 (1952), 248-253.

6. J. van Tiel, Espaces localement $K$-convexes $I, I I, I I I, I V$, Nederl. Akad. Wetensch. Proc. Ser. A 68 (1965), 249-289.

Received December 20, 1966. During work on this paper, the author held a National Science Foundation Graduate Fellowship.

HARVARD UNIVERSITY 


\section{PACIFIC JOURNAL OF MATHEMATICS}

\section{EDITORS}

\author{
H. ROYDEN \\ Stanford University \\ Stanford, California \\ R. R. Phelps \\ University of Washington \\ Seattle, Washington 98105
}

J. DUGUNDJI

Department of Mathematics

University of Southern California

Los Angeles, California 90007

RICHARD ARENS

University of California

Los Angeles, California 90024

\section{ASSOCIATE EDITORS}
E. F. BECKENBACH
B. H. NeumanN
F. WOLF
K. YosidA

\section{SUPPORTING INSTITUTIONS}

UNIVERSITY OF BRITISH COLUMBIA

CALIFORNIA INSTITUTE OF TECHNOLOGY

UNIVERSITY OF CALIFORNIA

MONTANA STATE UNIVERSITY

UNIVERSITY OF NEVADA

NEW MEXICO STATE UNIVERSITY

OREGON STATE UNIVERSITY

UNIVERSITY OF OREGON

OSAKA UNIVERSITY

UNIVERSITY OF SOUTHERN CALIFORNIA
STANFORD UNIVERSITY

UNIVERSITY OF TOKYO

UNIVERSITY OF UTAH

WASHINGTON STATE UNIVERSITY

UNIVERSITY OF WASHINGTON

AMERICAN MATHEMATICAL SOCIETY CHEVRON RESEARCH CORPORATION TRW SYSTEMS

NAVAL WEAPONS CENTER

Printed in Japan by International Academic Printing Co., Ltd., Tokyo, Japan 


\section{Pacific Journal of Mathematics}

\section{Vol. 26, No. $1 \quad$ November, 1968}

Efraim Pacillas Armendariz, Closure properties in radical theory......... 1

Friedrich-Wilhelm Bauer, Postnikov-decompositions of functors .......... 9

Thomas $\mathrm{Ru}-$ Wen Chow, The equivalence of group invariant positive definite

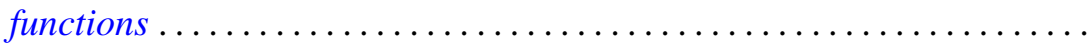

Thomas Allan Cootz, A maximum principle and geometric properties of

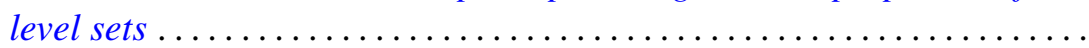

Rodolfo DeSapio, Almost diffeomorphisms of manifolds ............ 47

R. L. Duncan, Some continuity properties of the Schnirelmann density......

Ralph Jasper Faudree, Jr., Automorphism groups of finite subgroups of

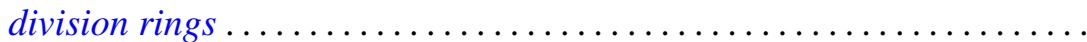

Thomas Alastair Gillespie, An invariant subspace theorem of $J$.

Feldman.........................................

George Isaac Glauberman and John Griggs Thompson, Weakly closed direct factors of Sylow subgroups .............................

Hiroshi Haruki, On inequalities generalizing a Pythagorean functional equation and Jensen's functional equation .....................

David Wilson Henderson, D-dimension. I. A new transfinite dimension.....

David Wilson Henderson, D-dimension. II. Separable spaces and

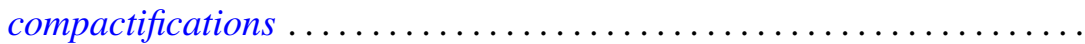

Julien O. Hennefeld, A note on the Arens products ............... 115

Richard Vincent Kadison, Strong continuity of operator functions ...

J. G. Kalbfleisch and Ralph Gordon Stanton, Maximal and minimal coverings of $(k-1)$-tuples by $k$-tuples.

Franklin Lowenthal, On generating subgroups of the Moebius group by pairs of infinitesimal transformations...

Michael Barry Marcus, Gaussian processes with stationary increments possessing discontinuous sample paths . .

Zalman Rubinstein, On a problem of Ilyeff ...

Bernard Russo, Unimodular contractions in Hilbert space. ...

David Lee Skoug, Generalized Ilstow and Feynman integrals...

William Charles Waterhouse, Dual groups of vector spaces . 